\title{
30c. SUB-COMMISSION ON THE OBSERVATION OF SPECTROSCOPIC BINARIES
}

This sub-commission was not reorganized immediately after the death of its former President, the late Dr J. H. Moore, and no report appeared in Vol. 8 of the Transactions (I954). However, the opinions of the remaining members of the sub-commission were favourable to its continuation, and accordingly it has now been reorganized. In so far as eclipsing binaries are also spectroscopic binaries, there will undoubtedly be a little overlapping of the functions of Sub-commission $30 \mathrm{c}$ and Commission 42. It is not anticipated that there will be extensive duplication of effort in connexion with this overlapping.

At several observatories the emphasis in spectrographic observation has gradually shifted away from pure radial velocity work and spectroscopic binary orbits to problems of a more definitely astrophysical nature. Even in the lists of stars of variable velocity that are now being followed, there are many for which the main purpose of observation is the study of phenomena other than, or in addition to, orbital motion. In the list that will be distributed directly to all members of Commission 30 and to all interested observatories, fully half of the objects fall in this latter category.

In spite of the shift of interest away from pure orbital motion, and doubtless in part because of the numerous special phenomena exhibited by binary systems, the number of stars of variable radial velocity now under observation is greater than ever. To a large extent this increase is due to the great growth of radial velocity work in the southern hemisphere. For the first time the list of binaries contains numerous objects that are being followed at Pretoria (both by Radcliffe and Cape astronomers), Cordoba and Canberra. The total number of entries in the list from these observatories is IIo.

Besides the list mentioned above, Dr Struve indicates that he is observing several stars of the $\beta$ Canis Majoris type, but the list of individual stars was not specifically given.

A list of fifty spectroscopic binaries and seventeen additional probable binaries discovered at Simeis has been received from Dr G. A. Shajn. The observation of these stars has not been resumed since its discontinuance during the war.

\author{
D. B. McLAughLIN \\ President of the Sub-commission
}

\section{Supplement to Draft Report}

\section{REPORT OF SUB-COMMISSION $30 a$ ON STANDARD Velocity STARS}

The General Catalogue of Stellar Radial Velocities, compiled by Dr R. E. Wilson in I953, lists a total of 2582 observations of the twenty-five bright standard velocity stars. Approximately one-fifth of the observations were secured with low-dispersion spectrographs, and the inclusion of such velocities of low weight weakens rather than strengthens the adopted velocities for the stars concerned. A thorough revision of the radial velocities has been undertaken, adopting the limiting dispersions of $22 \AA / \mathrm{mm}$. at $\mathrm{H} \gamma$ for the bright standard stars of Table I, and $38 \AA / \mathrm{mm}$. at $\mathrm{H} \gamma$ for the fainter stars of Table II.

Table I contains 2ro6 high-dispersion observations of the twenty-five stars, secured at fourteen different observatories, reduced to the Lick Observatory system by the application of the systematic corrections adopted by J. H. Moore (Lick Publications, vol. 18, 1932), and following his precepts regarding the relative weighting for the varying number of observations and the different dispersions employed. No systematic corrections were applied to the Lick or Victoria velocities. The recent high-dispersion observations at Victoria have been based upon the revised wave-length standards of R. M. Petrie (Contr. Dom. Ap. O. no. 4, I946, no. Io, I947, and no II, I948). 